\title{
Siderophore-based vaccine protects against $E$. coli infection
}

The emergence of antibiotic resistance poses a growing challenge to the management of patients with recurrent UTI, and effective alternatives to antibiotics are urgently needed. Now, researchers have developed a siderophorebased vaccine that prevents uropathogenic Escherichia coli (UPEC) infection.

These findings build upon previous research in this area, lead author Harry Mobley explains "We had developed an experimental vaccine in a mouse model of UTI. The outer membrane proteins that provided protection were receptors for small molecules called siderophores, which the bacteria use to scavenge iron from the host. We reasoned that we could also obtain an immune response if we conjugated these hydrophobic proteins to an adjuvant and used this product as a vaccine." First author Laura Mike explains the biological basis for targeting these small molecules "Siderophores strip iron from host stores, are recognized by protein receptors on the surface of the bacteria, and then internalized to deliver iron to the bacteria."

Researchers conjugated the siderophores yersiniabactin (Ybt) or aerobactin (Aer) to a cationized form of bovine serum albumin (cBSA). Mice were then immunized with either cBSA-Aer, cBSA-Ybt, a combination of cBSAYbt and cBSA-Aer, or cBSA alone as a control. Mice then underwent transurethral inoculation with UPEC strain HM69 at 21 days after the intial immunization, mice imunized with cBSAAer, cBSA-Ybt, or the combination had 19-fold, 12-fold, and 14-fold reductions in urinary bacterial burden, respectively, relative to that of controls at 48 hours after inoculation. Similar reductions in bacterial burden were observed in the kidneys of mice vaccinated with each agent alone; however, a more dramatic 126-fold reduction in bacterial burden was observed with use of both vaccines in combination. In order to confirm that the apparent protective effects of these vaccinations is dependent upon

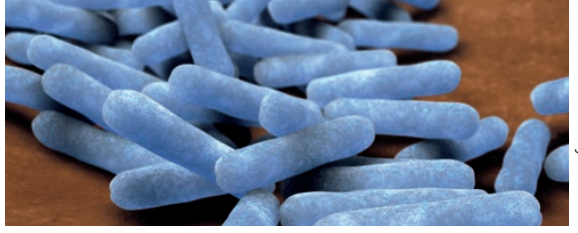

an immune response to these specific interactions, mice immunized against the wild type forms of Aer or Ybt were then inoculated with UPEC with mutated $y b t S$ or iuc4 (encoding the Ybt or Aer siderophores, respectively): no significant changes in bacterial load were observed in any of these immunized mice.

These data confirm the potential of siderophore-based vaccines as an alternative to antibiotics for the prevention of UTI, however, Mobley highlights that further research is clearly required "We can use combinations of the antigens, modify the route of administration and use adjuvants approved for use in humans. Our goal would be to improve the efficacy (or effectiveness) of the next generation vaccine."

Peter Sidaway

ORIGINAL ARTICLE Mike, L. A. et al. Siderophore vaccine conjugates protect against uropathogenic Escherichia coli urinary tract infection. Proc. Natl Acad. Sci. USA http://dx.doi.org/10.1073/pnas.1606324113 (2016) 